\title{
ON THE SURJECTIVITY CRITERION FOR BUCHSBAUM MODULES
}

\author{
SHIRO GOTO \\ (Communicated by Louis J. Ratliff, Jr.) \\ Dedicated to Professor Hideyuki Matsumura on his 60th birthday
}

\begin{abstract}
Let $R$ be a Cohen-Macaulay local ring with maximal ideal $m$ and suppose that $\operatorname{dim} R \geq 2$. Then $R$ is regular if (and only if) for any Buchsbaum $R$-module $M$ and for any integer $i, i \neq \operatorname{dim}_{R} M$, the canonical map $\operatorname{Ext}_{R}^{i}(R / m, M) \rightarrow H_{m}^{i}(M):=\lim _{\vec{n}} \operatorname{Ext}_{R}^{i}\left(R / m^{n}, M\right)$ is surjective. The hypothesis that $R$ is Cohen-Macaulay is not superfluous. Two examples are given.
\end{abstract}

\section{INTRODUCTION}

The purpose of this paper is to prove the following

Theorem 1.1. Let $R$ be a Cohen-Macaulay local ring with maximal ideal $\mathfrak{m}$ and suppose that $\operatorname{dim} R \geq 2$. Then the following two conditions are equivalent.

(1) $R$ is a regular local ring.

(2) For any Buchsbaum $R$-module $M$ and for any integer $i \neq \operatorname{dim}_{R} M$, the canonical map

$$
\operatorname{Ext}_{R}^{i}(R / \mathfrak{m}, M) \stackrel{\varphi_{M}^{i}}{\rightarrow} H_{\mathrm{m}}^{i}(M):=\underset{n}{\lim } \operatorname{Ext}_{R}^{i}\left(R / \mathfrak{m}^{n}, M\right)
$$

is surjective.

In the above theorem our contribution is the implication $(2) \Rightarrow(1)$ and the reverse one is due to J. Stückrad [4, Satz 2].

As is well known, Stückrad and Vogel discovered in 1978 a cohomological criterion, so-called now the surjectivity criterion for Buchsbaum modules:

Surjectivity criterion ([4, Satz 2] and [5, p. 732, Theorem 1]). Let $M$ be a finitely generated module over a Noetherian local ring $(R, \mathfrak{m})$. If the canonical

Received by the editors February 13, 1989 and, in revised form, May 19, 1989.

1980 Mathematics Subject Classification (1985 Revision). Primary 13H05, 13H10; Secondary $13 \mathrm{H} 15$.

Partially supported by Grant-in-Aid for Co-operative Research. 
$\operatorname{map} \operatorname{Ext}_{R}^{i}(R / \mathfrak{m}, M) \stackrel{\varphi_{M}^{i}}{\rightarrow} H_{\mathrm{m}}^{i}(M)$ is surjective for any $i \neq \operatorname{dim}_{R} M, M$ is a Buchsbaum $R$-module. When $R$ is regular, the converse is also true.

This criterion is general enough and really powerful. In fact, passing to the m-adic completion $\widehat{R}$ of $R$ and appealing to the structure theorem of Cohen, one may assume the base ring $R$ to be regular; hence a given $R$-module $M$ is Buchsbaum if and only if the maps $\varphi_{M}^{i}$ are surjective for all $i \neq \operatorname{dim}_{R} M$. Comparing with this clear assertion one might feel our Theorem (1.1) somewhat pedantic. However there has been known only one example of Buchsbaum modules $M$ which fail to have the surjectivity of the maps $\varphi_{M}^{i}$, provided that $R$ is not regular (cf. [4]). On the contrary Theorem (1.1) and its proof claim that any nonregular Cohen-Macaulay local ring $R$ of $d=\operatorname{dim} R \geq 2$ possesses at least one Buchsbaum $R$-module $M$ of $\operatorname{dim}_{R} M=d$, for which the canonical $\operatorname{map} \operatorname{Ext}_{R}^{1}(R / \mathfrak{m}, M) \stackrel{\varphi_{M}^{1}}{\rightarrow} H_{\mathrm{m}}^{1}(M)$ is not surjective.

The proof of Theorem (1.1) shall be given in the next section. Unfortunately the hypothesis in (1.1) that $R$ is Cohen-Macaulay cannot be removed. There exists a nonregular Buchsbaum local ring $R$ of $\operatorname{dim} R=2$ that satisfies the condition (2) of (1.1) (cf. Proposition (3.2)). We will explore two examples in $\S 3$.

Throughout this paper let $R$ stand for a Noetherian local ring with maximal ideal $\mathrm{m}$ and let $H_{\mathrm{m}}^{i}(\cdot)$ denote the $i$ th local cohomology functor relative to $\mathrm{m}$.

\section{Proof of Theorem 1.1}

In this section we assume that $R$ is a Cohen-Macaulay ring of $d=$ $\operatorname{dim} R \geq 2$. We choose a minimal system $x_{1}, x_{2}, \ldots, x_{n}$ of generators for the maximal ideal $\mathrm{m}$ so that the sequence $x_{i_{1}}, x_{i_{2}}, \ldots, x_{i_{d}}$ forms a system of parameters of $R$ for any $1 \leq i_{1}<i_{2}<\cdots<i_{d} \leq n$. Let

$$
0 \longrightarrow L \longrightarrow R^{n} \stackrel{\left[x_{1} x_{2} \cdots x_{n}\right]}{\longrightarrow} R \longrightarrow R / \mathfrak{m} \longrightarrow 0
$$

denote the initial part of a minimal free resolution of $R / \mathfrak{m}$ and let $\left\{e_{i}\right\}_{1 \leq i \leq n}$ be the standard basis of $R^{n}$. Then $L \ni f_{i j}:=x_{i} e_{j}-x_{j} e_{i}(1 \leq i<j \leq n)$. We denote by $K$ the $R$-submodule of $L$ generated by the Koszul relations $\left\{f_{i j}\right\}_{1 \leq i<j \leq n}$. Let

$$
N=\mathfrak{m} L+K \quad \text { and } \quad M=R^{n} / N .
$$

Then we have

Proposition 2.1. $M$ is a Buchsbaum R-module of $\operatorname{dim}_{R} M=d$ and

$$
\begin{aligned}
H_{\mathrm{m}}^{i}(M) & =L / N & & (i=0), \\
& =R / \mathfrak{m} & & (i=1), \\
& =(0) & & (i \neq 0,1, d) .
\end{aligned}
$$


First let us give a proof of (1.1) modulus (2.1). It suffices to prove the implication $(2) \Rightarrow(1)$. Because $\operatorname{Ext}_{R}^{i}(R / \mathfrak{m}, M) \stackrel{\varphi_{M}^{i}}{\rightarrow} H_{\mathrm{m}}^{i}(M)$ is surjective for any $i \neq d$, we get by [5, p. 734, Lemma 6] that the homomorphism

$$
j_{*}: \operatorname{Ext}_{R}^{2}(R / \mathfrak{m}, L / N) \rightarrow \operatorname{Ext}_{R}^{2}(R / \mathfrak{m}, M)
$$

induced from the imbedding $H_{\mathrm{m}}^{0}(M)=L / N \stackrel{j}{\rightarrow} M$ is injective. Let

$$
\cdots \rightarrow F_{3} \stackrel{\partial_{3}}{\rightarrow} F_{2} \stackrel{\partial_{2}}{\rightarrow} F_{1}=R^{n} \stackrel{\partial_{1}=\left[x_{1} x_{2} \cdots x_{n}\right]}{\longrightarrow} F_{0}=R \rightarrow R / \mathfrak{m} \rightarrow 0
$$

denote a minimal free resolution of $R / \mathfrak{m}$ and recall that the map

$$
j_{*}: \operatorname{Ext}_{R}^{2}(R / \mathfrak{m}, L / N) \rightarrow \operatorname{Ext}_{R}^{2}(R / \mathfrak{m}, M)
$$

is induced from the following homomorphism

$$
\begin{aligned}
& \cdots \rightarrow \operatorname{Hom}_{R}\left(F_{1}, L / N\right) \stackrel{\partial_{2}^{*}}{\rightarrow} \operatorname{Hom}_{R}\left(F_{2}, L / N\right) \stackrel{\partial_{3}^{*}}{\rightarrow} \operatorname{Hom}_{R}\left(F_{3}, L / N\right) \rightarrow \ldots \\
& \downarrow j_{*} \quad \downarrow j_{*} \quad \downarrow j_{*} \\
& \cdots \rightarrow \operatorname{Hom}_{R}\left(F_{1}, M\right) \underset{\partial_{2}^{*}}{\longrightarrow} \operatorname{Hom}_{R}\left(F_{2}, M\right) \underset{\partial_{3}^{*}}{\longrightarrow} \operatorname{Hom}_{R}\left(F_{3}, M\right) \rightarrow \ldots
\end{aligned}
$$

of complexes. Consider the commutative diagram

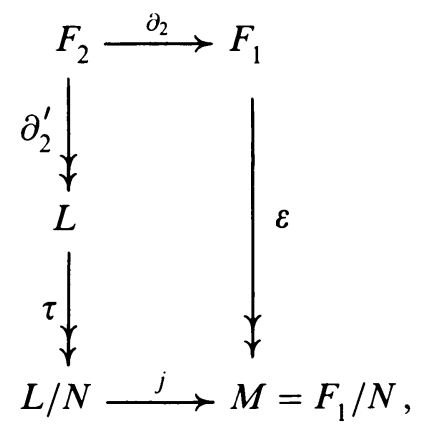

where $\varepsilon, \tau$ are the canonical epimorphisms and $\partial_{2}^{\prime}$ denotes the epimorphism induced from $\partial_{2}$. Then the cohomology class $\overline{\tau \circ \partial_{2}^{\prime}}$ of $\tau \circ \partial_{2}^{\prime}$ is contained in the kernel of $\operatorname{Ext}_{R}^{2}(R / \mathfrak{m}, L / N) \stackrel{j_{*}}{\rightarrow} \operatorname{Ext}_{R}^{2}(R / \mathfrak{m}, M)$. Because $\operatorname{Hom}_{R}\left(F_{2}, L / N\right)=$ $\operatorname{Ext}_{R}^{2}(R / \mathfrak{m}, L / N)$ and the homomorphism $j_{*}$ is injective, we see $\tau \circ \partial_{2}^{\prime}=$ $\overline{\tau \circ \partial_{2}^{\prime}}=0$ whence $L=N$. As $N=\mathfrak{m} L+K$ by definition, we get $L=K$, that is the module $L$ of the relations of the minimal system $x_{1}, x_{2}, \ldots, x_{n}$ of generators for $m$ is generated by the Koszul relations $\left\{x_{i} e_{j}-x_{j} e_{i}\right\}_{1 \leq i<j \leq n}$. Thus $R$ has to be regular (by an easy Koszul argument: $H_{1}\left(x_{1}, x_{2}, \ldots, x_{n} ; R\right)=(0)$ if and only if $x_{1}, x_{2}, \ldots, x_{n}$ is an $R$-regular sequence).

Proof of Proposition 2.1. By the short exact sequence $0 \rightarrow L / N \rightarrow M \rightarrow \mathfrak{m} \rightarrow$ 0 , we get the second assertion. Hence $M$ is a generalized Cohen-Macaulay $R$-module, that is the length $l_{R}\left(H_{\mathrm{m}}^{i}(M)\right)$ of $H_{\mathrm{m}}^{i}(M)$ is finite for any $i \neq$ $\operatorname{dim}_{R} M$, and $I_{R}(M)=l_{R}(L / N)+(d-1)$ (cf., e.g., [6,7]). To prove that $M$ is Buchsbaum we need the following lemma. 
Lemma 2.2 [7, Proposition 3.2]. Let $R$ be a Noetherian local ring and let $M$ be a generalized Cohen-Macaulay $R$-module. Then $M$ is Buchsbaum if and only if the maximal ideal $\mathfrak{m}$ of $R$ contains a system $x_{1}, x_{2}, \ldots, x_{n}$ of generators that satisfies the following condition: For any $1 \leq i_{1}<i_{2}<\cdots<i_{s} \leq n$ $\left(s=\operatorname{dim}_{R} M\right)$, the elements $x_{i_{1}}, x_{i_{2}}, \ldots, x_{i_{s}}$ form a system of parameters for $M$ and one has the equality

$$
l_{R}(M / \mathfrak{q} M)-e_{\mathfrak{q}}(M)=I_{R}(M)
$$

where $\mathfrak{q}=\left(x_{i_{1}}, x_{i_{2}}, \ldots, x_{i_{s}}\right) R$.

Let $1 \leq i_{1}<i_{2}<\cdots<i_{d} \leq n$ be integers and put $\mathfrak{q}=\left(x_{i_{1}}, x_{i_{2}}, \ldots, x_{i_{d}}\right) R$. Then by virtue of (2.2), because our module $M$ is generalized Cohen-Macaulay and $\operatorname{dim}_{R} M=d$, we have only to see the equality

$$
l_{R}(M / \mathfrak{q} M)-e_{\mathfrak{q}}(M)=l_{R}(L / N)+(d-1) .
$$

Recall that the maximal ideal $\mathfrak{m}$ is a Buchsbaum $R$-module of $I_{R}(\mathfrak{m})=d-1$ (cf. [1, Proposition (2.4)]). Then as $e_{\mathfrak{q}}(M)=e_{\mathfrak{q}}(\mathfrak{m})$, we have

$$
\begin{aligned}
l_{R}(M / \mathfrak{q} M)-e_{\mathfrak{q}}(M) & =l_{R}(M / \mathfrak{q} M)-e_{\mathfrak{q}}(\mathfrak{m}) \\
& =l_{R}(M / \mathfrak{q} M)-\left[l_{R}(\mathfrak{m} / \mathfrak{q m})-(d-1)\right] \\
& =\left[l_{R}(M / \mathfrak{q} M)-l_{R}(\mathfrak{m} / \mathfrak{q m})\right]+(d-1) .
\end{aligned}
$$

Consequently, in order to prove that $M$ is Buchsbaum, it is enough to check the equality

$$
l_{R}(M / \mathfrak{q} M)=l_{R}(L / N)+l_{R}(\mathfrak{m} / \mathfrak{q m})
$$

that is the sequence

$$
0 \rightarrow L / N \rightarrow M / \mathfrak{q} M \rightarrow \mathfrak{m} / \mathfrak{q} \mathfrak{m} \rightarrow 0
$$

induced from the short exact sequence $0 \rightarrow L / N \rightarrow M \rightarrow \mathfrak{m} \rightarrow 0$ remains exact, or equivalently

$$
L \cap \mathfrak{q} \cdot R^{n} \subset N
$$

which immediately follows from the next

Claim (2.3). $L \cap q \cdot R^{n} \subset K$.

Proof of Claim 2.3. We may assume $\mathfrak{q}=\left(x_{1}, x_{2}, \ldots, x_{d}\right) R$. As

$$
\mathfrak{q} \cdot R^{n} \subset \sum_{i=1}^{d} \operatorname{Re}_{i}+K
$$

it suffices to show that

$$
L \cap \sum_{i=1}^{d} \operatorname{Re}_{i} \subset K .
$$

Let

$$
v=\sum_{i=1}^{d} a_{i} e_{i} \in L
$$


and we have $\sum_{i=1}^{d} a_{i} x_{i}=0$. Because $x_{1}, x_{2}, \ldots, x_{d}$ is an $R$-regular sequence, we see

$$
\sum_{i=1}^{d} a_{i} e_{i}=\sum_{1 \leq i<j \leq d} b_{i j}\left(x_{i} e_{j}-x_{j} e_{i}\right)
$$

for some $b_{i j} \in R$ which means $v \in K$ as required. This completes the proof of Theorem (1.1) as well as (2.3).

\section{Counterexamples}

Let $M$ be a Buchsbaum $R$-module of $\operatorname{dim}_{R} M=s$ and $\operatorname{depth}_{R} M=t$. Then it is easy to check that the canonical map $\operatorname{Ext}_{R}^{t}(R / \mathfrak{m}, M) \stackrel{\varphi_{M}^{t}}{\rightarrow} H_{\mathrm{m}}^{t}(M)$ is an isomorphism if $t<s$ (cf. [5, p. 736, Corollary 1.1]). Accordingly, whenever $t<s$ and $H_{\mathrm{m}}^{i}(M)=(0)$ for all $i \neq t, s$, the Buchsbaum $R$-module $M$ enjoys the surjectivity property (2) stated in (1.1). This is the reason why in Theorem (1.1) we have assumed that $\operatorname{dim} R \geq 2$. By the same reason we see that in case $\operatorname{dim} R=2$, the ring $R$ satisfies the condition (2) of (1.1) if and only if any Buchsbaum $R$-module $M$ of $\operatorname{dim}_{R} M=2$ enjoys the surjectivity property (2) in (1.1).

Proposition 3.1. Let $R$ be a two-dimensional local integral domain of $e(R)=1$. Then $R$ satisfies the condition (2) of (1.1).

Proof. By [4, Satz 2] we may assume that $R$ is nonregular. Then $R$ possesses no Buchsbaum module $M$ of $\operatorname{dim}_{R} M=2$. In fact, assume the contrary and choose a Buchsbaum $R$-module $M$ of $\operatorname{dim}_{R} M=2$. Then as $R$ is an integral domain, $R$ is contained in the endomorphism algebra $\operatorname{End}_{R} M$ whence $\widehat{R}$ is a subalgebra of $\operatorname{End}_{\widehat{R}} \widehat{M}$. Let $\mathfrak{P} \in$ Ass $\widehat{R}$. Then as $\mathfrak{P} \in \operatorname{Ass}_{\widehat{R}} \widehat{M}$ and as $\widehat{M}$ is a Buchsbaum $\widehat{R}$-module, we have either $\operatorname{dim} \widehat{R} / \mathfrak{P}=2$ or $\mathfrak{P}=\mathfrak{m} \widehat{R}$ (cf. [5, p. 730, Lemma 2]). Of course, since depth $\widehat{R}>0$, we get $\mathfrak{P} \neq \mathrm{m} \widehat{R}$ and so $\operatorname{dim} \widehat{R} / \mathfrak{P}=2$ for any $\mathfrak{P} \in$ Ass $\widehat{R}$. Hence $R$ is unmixed, which implies by [3, (40.6) Theorem] that $R$ is a regular local ring because $e(R)=1$ by our assumption-this contradicts the choice of $R$. Thus $R$ possesses no Buchsbaum module $M$ of $\operatorname{dim}_{R} M=2$.

In his famous book [3, p. 203, Example 2] Nagata constructed a twodimensional nonregular local integral domain $R$ of $e(R)=1$. His example asserts by (3.1) that the hypothesis in (1.1) that $R$ is Cohen-Macaulay is not superfluous.

Proposition 3.2. Let $S$ be a three-dimensional regular local ring with maximal ideal $\mathfrak{n}$. Let $X \in \mathfrak{n} \backslash \mathfrak{n}^{2}$ and let $I$ be a proper ideal in $S$ of $h t_{S} I \geq 2$. Then the ring $R=S / X I$ satisfies the condition (2) in (1.1).

Proof. Let $\mathfrak{P}=X R$. Then $R$ possesses exactly three isomorphism classes of indecomposable Buchsbaum $R$-modules $M$ of $\operatorname{dim}_{R} M(=\operatorname{dim} R)=2$ and the 
$R$-modules

$$
M_{0}=R / \mathfrak{m P}, \quad M_{1}=\mathfrak{m} / \mathfrak{P}, \quad \text { and } M_{2}=R / \mathfrak{P}
$$

are the representatives of them (cf. [2, Theorem (3.1)]). Since $H_{\mathfrak{m}}^{i}\left(M_{j}\right)=0$ if $i \neq j, 2$, the $R$-modules $M_{j}(j=0,1,2)$ enjoy the property (2) in (1.1). Because any Buchsbaum $R$-module $M$ of $\operatorname{dim}_{R} M=2$ is isomorphic to a direct sum of $M_{j}$ 's together with a vector space over $R / \mathfrak{m}$, we see that $M$ has the required property (2) stated in (1.1). Thus $R$ satisfies the condition (2) of (1.1).

In the above proposition, if we choose $I=\mathfrak{n}, R=S / X \mathfrak{n}$ is a nonregular Buchsbaum ring. This example shows that the hypothesis in (1.1) that $R$ is Cohen-Macaulay cannot be replaced by the weaker one that $R$ is Buchsbaum.

\section{REFERENCES}

1 S. Goto, On Buchsbaum rings, J. Algebra 67 (1980), 272-279.

2 _ Commutative Algebra (edited by M. Hochster, C. Huneke, and J. D. Sally), Proceedings of a Microprogram held June 15-July 2, 1987, Springer-Verlag (1989), 247-263.

3 M. Nagata, Local rings, Interscience, New York, 1962.

4 J. Stückrad, Über die kohomologische Charakterisierung von Buchsbaum-Moduln, Math. Nachr. 95 (1980), 265-272.

5. J. Stückrad and W. Vogel, Toward a theory of Buchsbaum singularities, Amer. J. Math. 100 (1978), 727-746.

6 N. Suzuki, Canonical duality for unconditioned strong $d$-sequences, J. Math. Kyoto Univ. 26 (1986), 571-593.

7 N. V. Trung, Toward a theory of generalized Cohen-Macaulay modules, Nagoya Math. J. 102 (1986), 1-49.

Department of Mathematics, Nihon University, College of Humanities and Sciences, TOKYO 156, JAPAN 